\title{
Caregiver responses to symptoms of first-onset psychosis: A comparative study of Chinese-and Euro-Canadian families
}

\author{
Andrew G. Ryder ${ }^{1}$, Bean Graham ${ }^{2}$, and Kenneth L. Dion ${ }^{1}$ \\ ${ }^{1}$ University of Toronto \\ ${ }^{2}$ Clarke Institute of Psychiatry
}

\begin{abstract}
Abstract Considerable evidence suggests that ethnic Chinese families in Canada are reluctant to seek psychiatric treatment for a relative with mental illness. The following hypotheses were investigated: (a) longer delay in seeking treatment among Chinese versus Euro-Canadians; (b) greater burden among Chinese versus Euro-Canadians; and (c) more negative conceptions of mental illness among Chinese versus Euro-Canadians. The sample consisted of 18 Chinese Canadians and 36 Euro-Canadians experiencing a first episode of psychosis; each participant recruited a parent or sibling who responded to measures of perceived family burden and attitudes toward mental illness. Results confirmed all three hypotheses. Chinese care-givers were particularly more likely to endorse the practice of keeping mental illness a secret from others, as well as withdrawal from individuals with mental illness. These findings suggest that Chinese caregivers were more affected by the stigma of mental illness than were Euro-Canadian caregivers.
\end{abstract}

Keywords: caregiver-burden, Chinese, coping-behaviour, mental health services, treatment-seeking

The schizophrenias are a group of psychotic disorders which occur in virtually all societies, and are characterized primarily by cognitive disorganization, gross distortions of reality, and social withdrawal. Many individuals who develop schizophrenia rely on their parents and other close family members for support, as these disorders have a median age of onset for a first psychotic episode in the earlyto-mid-20s for men and the late 20s for women (American Psychiatric Association, 1994). Since the majority of deinstitutionalized persons with mental illness live with their families, with the vast majority being adults who live with their parents (Aviram, 1990), it is important for researchers to study the experience of schizophrenia within the context of the family, exploring the ways in which family life affects, and is affected by, this disorder.

The presence of the symptoms of schizophrenia, combined with the stigma surrounding mental illness, can lead to considerable stress for the individual and his or her family. Families experience stress as a consequence of having a family member with mental illness (Hoenig \& Hamilton, 1965; MacCarthy et al., 1989), with significant burden typically being experienced regardless of whether or not the individual with mental illness is living with his or her family (Hatfield, 1986). This factor, termed emphfamily burden in the literature, is a potentially important variable in the interaction between the individual with mental illness and his or her family. For example, a highly burdened family may be less able to provide the necessary level of caregiving. However, while there is considerable cross-cultural evidence demonstrating that family life may exert a significant influence on the course of schizophrenia (e.g. Smith, Birchwood, Cochrane, \& George, 1992), the economic and human burdens facing care-givers have not been extensively studied thus far (Lefley, 1987; Loukissa, 1995) and the specific determinants of burden are not yet well understood (Mueser, Webb, Pfeiffer, Gladis, \& Levinson, 1996).

Hoenig and Hamilton (1965) found that $60 \%$ of caregivers reported burden as a result of the disturbed family member's acting out, lack of self- care, reclusiveness and/or need for close supervision. In order to separate observable evidence of burden from emotional reactions to caregiving, they classified worrisome behaviours and disruptions in family life as emphobjective burden and other experiences of stress as emphsubjective burden. Both forms of burden have been positively associated with symptom severity, with a stronger relationship found for objective burden (Siegel, Raveis, Mor, \& Houts, 1991).

Given that people of different cultural backgrounds may differ in terms of family and community structures, attitudes towards mental health and illness, and behavioural norms, there is reason to expect that the correlates of family burden might differ among various ethnic groups. Unfortunately, the potential role of ethnicity as a correlate of family burden has been largely ignored in the literature. However, a substantial 
body of research exploring cross-cultural differences in mental health and illness exists, with a particular focus on those ethnic groups that form sizable minorities in North America (Yeh, Takeuchi, \& Sue, 1994). One such group is the Chinese (Dion \& Dion, 1996).

As in other cultures, ideas about health and disease among the Chinese are based on socioculturally determined beliefs and values. Furthermore, there are also cross-cultural differences in definitions of how a family is constituted, what the family's responsibility is for the care of ill members, which family members are chiefly responsible for this care, and the role of the extended family (Rolland, 1987). In particular there is evidence to suggest that, within Chinese culture, mental illness is highly stigmatic for the entire family (Lin \& Lin, 1981; Shon \& Ja, 1982), resulting in efforts to control the illness within the family for as long as possible (Kirmayer, 1989; Sue \& McKinney, 1975). This pattern might then be expected to influence family burden and treatment delay among Chinese individuals with psychotic mental illness living in North America.

Considerable research has established that having a family member with mental illness can lead to high levels of stress and burden for caregivers and several cultural studies of Chinese families and mental health have suggested that mental illness often results in particularly high levels of stigma and distress for family members (e.g. Lin \& Lin, 1981; Shon $\& \mathrm{Ja}, 1982)$. We thus explored the possibility that Chinese or Euro-Canadian ethnicity may be an important correlate of the experience of mental illness by family members. Specifically, we predicted that Chinese ethnicity would be associated with (a) longer latencies between the onset of symptoms and initiation of treatment compared to Euro-Canadians, (b) greater perceived burden among Chinese families compared to Euro-Canadian families, and (c) a more negative conceptualization of mental illness compared to Euro- Canadian families.

\section{Method}

\section{Participants}

\section{Recruitment}

The data for this study were obtained from an investigation of first-onset psychosis conducted at the Clarke Institute of Psychiatry in Toronto, Canada from 1992 to 1995 by Graham Bean and Jiahui Zhang-Wong. The First-Onset Schizophrenia Project recruited individuals from the greater Toronto Area experiencing a first lifetime episode of psychosis. Respondents met inclusion criteria if they: (a) were between 18 and 54 years of age, (b) were experiencing a first lifetime episode of psychosis, (c) had not received psychotropic medication prior to the illness episode, and (d) had no evidence of prior or current cerebral disease, mental retardation, chronic physical illness, or substance dependence.
Each participant was asked to recruit one of their caregivers, preferably a parent or sibling, to take part in the project.

\section{Demographic Characteristics}

The Chinese group comprised 9 males and 9 females with a mean age of 25.3 years, while the Euro-Canadian group comprised 24 males and 12 females with a mean age of 26.0 years. Socioeconomic status of the immediate family was measured using composite SES scores derived from Blishen, Carroll, and Moore's (1987) index of occupations in Canada, yielding scores of 44.0 for the Chinese group and 52.5 for the Euro-Canadian group. SES scores were based on the occupation of the primary wage-earner, usually a parent. Chisquare revealed no significant differences for gender, and $t$ tests were non-significant for age and SES. However, the results for these analyses should be interpreted with caution due to the low power associated with small sample sizes. (Note that we ran $2 \times 2$ ANCOVAs with ethnicity and gender as independent variables and SES as a covariate for all analyses in the results, and found that all of the previously significant effects remained significant, $p<.05$; gender, ethnicity $\mathrm{X}$ gender, and the covariate term never attained significance at the $p<.05$ level.)

There were no significant differences between the Chinese and Euro-Canadian groups on either positive or negative symptoms (Zhang-Wong, Beiser, Zipursky, \& Bean, 1998). Most participants received a diagnosis of schizophrenia except for two with schizophreniform disorder, one with schizoaffective disorder, and one with delusional disorder in the Chinese group, and five with delusional disorder, four with schizophreniform disorder, two with atypical psychosis, and one with schizoaffective disorder in the Euro-Canadian group. Although most of the data for this study were obtained prior to treatment, some variables were assessed by a questionnaire mailed to the families after treatment completion. Because only 29 of the 54 participants returned the questionnaire, the possibility of selective loss between the two ethnic groups was investigated. Thirty-nine percent of the Chinese group and $61 \%$ of the Euro-Canadian group returned the package, which was suggestive but not statistically significant, $\chi^{2}=2.38, p=.12$.

\section{Procedure and Measures}

After obtaining informed consent, a trained Master's level research assist- ant collected demographic and psychosocial information from the participants. At intake, a psychiatrist or clinical psychologist conducted a diagnostic interview using the SCID (Spitzer, Williams, \& Gibbon, 1987) and gathered information on premorbid history and progress of the illness. Participants also completed the Onset Questionnaire (Beiser, Erickson, Fleming, \& Iacono, 1993). After the completion of treatment, caregivers were mailed the 24-item Perceived Family Burden Scale (PFBS; Levene, Lancee, \& See- 
man, 1996), and a 42-item questionnaire exploring attitudes towards the mentally ill (including items from Link, 1987; Link, Mirotznik, \& Cullen 1991).

\section{Onset Questionnaire}

The duration of untreated illness prior to admission was assessed by the Onset Questionnaire (Beiser et al., 1993), which uses a checklist of words and phrases indicative of both the first noticeable signs of deterioration and the manifestation of psychotic symptoms. Two raters used this information to assign durations for prodrome (difference between first noticeable signs and onset of prominent psychotic symptoms) and treatment delay (difference between onset of prominent psychotic symptoms and initiation of medical or traditional treatment seeking). As is often the case with latency scores, these measures were positively skewed and thus were logarithmically transformed before further analysis (see Howell, 1992, p. 311).

\section{Perceived Family Burden Scale}

The PFBS (Levene et al., 1996) was developed to examine the caregiver's perception of the extent to which various behaviours associated with psychosis resulted in burden for the family. This scale contains a list of 24 such behaviours, which are rated as present or absent. Each present behaviour is also rated for the extent to which the caregiver is burdened by its occurrence. Thus, the former looks at objective burden, whereas the latter focuses on subjective burden, as defined by Hoenig and Hamilton (1965). Each item is rated on a scale of 0 to 4 , in which 0 represents absence of the behaviour, 1 represents presence of the behaviour but absence of burden, and 2, 3, and 4 represent mild, moderate, and severe burden, respectively. The scale had very high internal consistency in the present sample with Cronbach's alpha $=.90$.

\section{Attitudes Towards the Mentally Ill}

As noted earlier, participants completed a 42-item followup questionnaire designed to investigate attitudes towards individuals with mental illness. All items were scored in the negative direction on a 6-point Likert scale, and a total score was generated from the mean of the items. Because the participant-item ratio was insufficient for factor analysis, rational criteria were used to devise several subscales for exploratory purposes, as follows: (a) devaluation (beliefs that persons with mental illness are inferior), (b) blame (tendencies to blame individuals for their mental illness), (c) secret (the extent to which one would attempt to conceal an association with an individual who has a mental illness), (d) nonsupport (unwillingness to provide care and assistance to a person with mental illness), and (e) withdrawal (tendencies to reduce contact with associates who have a mental illness). The first three subscales were derived from scales designed by Link (1987; Link et al., 1991), whereas the last two were developed specifically for the first-onset project. The total score, and each of the five subscales, yielded acceptable coefficient alpha values: total, .92, devaluation, .88, blame, .87, secret, .86, non-support, .80, and withdrawal, .75 .

\section{Results}

\section{Latency Measures}

Individual latency measures were analyzed with univariate ANOVAs as a function of ethnicity. Treatment delay after onset of psychotic symptoms was significantly longer for Chinese participants than Euro-Canadian participants, $F(1,50)=$ $4.12, p<.05$. Group differences for prodrome were not significant, $F(1,50)=1.96$, $n s$ (see first panel of Table 1). Due to unequal cells and variances, estimated power could not be computed for prodrome (Cohen, 1988).

\section{Perceived Family Burden Scale}

Looking first at objective burden, Chinese caregivers reported more present behaviours than did Euro-Canadian participants, $F(1,27)=6.57, p<.02$. Decomposing this result revealed that Chinese participants reported significantly more burdensome behaviours, $F(1,27)=6.01, p<$ .03 , but the two groups did not significantly differ for nonburdensome behaviours, $F(1,27)=1.98$, ns. For subjective burden, Chinese participants reported a higher overall mean score than did Euro-Canadian participants, $F(1,27)=6.89, p$ $<$.02. However, there were no longer significant group differences when only present behaviours were considered as a component of the mean score, $F(1,27)=0.16$, $n$ s (see second panel of Table 1). However, it should be noted that due to the small sample size, this test had low power $(<.20)$ to detect effects found to be non-significant.

\section{Attitudes Towards the Mentally Ill}

Chinese participants' overall attitudes towards the mentally ill were more negative than were those of the EuroCanadian participants, $F(1,27)=4.93, p<.04$. Previously, this questionnaire was divided into five exploratory subscales which were then analyzed using univariate ANOVA. The Bonferroni correction was applied to control for alpha slippage, thus $p<.01$ (i.e. .05/5) was required for statistical significance. Chinese participants scored significantly higher than Euro-Canadian participants on the secret and withdrawal subscales, $F \mathrm{~s}(1,27)=10.78$ and $8.00, p \mathrm{~s}=.003$ and .009, respectively (see third panel of Table 1). Again, however, this test had low power $(<.20)$ to detect effects found to be non-significant.

\section{Discussion}

Each of the three research hypotheses were confirmed in this study. First, we found that Chinese individuals with 
Table 1

Descriptive Statistics for Latency, Family Burden, and Attitude Measures.

\begin{tabular}{|c|c|c|c|c|}
\hline \multirow[t]{2}{*}{ Measure } & \multicolumn{2}{|c|}{ Chinese } & \multicolumn{2}{|c|}{ Euro-Canadian } \\
\hline & Mean & $S D$ & Mean & $S D$ \\
\hline \multicolumn{5}{|l|}{ Panel 1 - Latency Measures } \\
\hline Prodrome in days (Log) & 2.41 & 0.81 & 1.97 & 1.17 \\
\hline Treatment Delay in days $(\mathrm{Log})^{*}$ & 2.22 & 0.69 & 1.77 & 0.78 \\
\hline \multicolumn{5}{|l|}{ Panel 2 - Perceived Family Burden } \\
\hline Number Behaviours Reported* & 17.86 & 8.17 & 10.59 & 5.98 \\
\hline Number Burdensome Behaviours Reported ${ }^{\star}$ & 15.14 & 6.89 & 9.73 & 4.44 \\
\hline Number Non-Burdensome Behaviours Reported & 2.71 & 4.42 & 0.86 & 2.49 \\
\hline Mean Total Burden** & 2.13 & 0.95 & 1.27 & 0.68 \\
\hline Mean Burden for Present Behaviours & 3.04 & 0.68 & 2.95 & 0.47 \\
\hline \multicolumn{5}{|l|}{ Panel 3 -Attitudes Towards Mental Illness } \\
\hline Total $^{*}$ & 4.00 & 0.54 & 3.35 & 0.71 \\
\hline Blame & 3.21 & 1.17 & 2.90 & 1.03 \\
\hline Devaluation & 4.36 & 1.00 & 3.98 & 0.93 \\
\hline Non-Support & 2.86 & 0.77 & 2.58 & 1.02 \\
\hline Secret $^{* *}$ & 5.02 & 1.00 & 3.62 & 0.98 \\
\hline Withdrawal ${ }^{* *}$ & 4.71 & 0.78 & 3.60 & 0.94 \\
\hline
\end{tabular}

$* p<.05 . * * p<.01$

a first onset of psychosis took longer to seek treatment as compared to Euro- Canadians after the onset of prominent psychotic symptoms. Second, Chinese caregivers reported a higher number of burdensome behaviours compared with Euro-Canadian caregivers, as well as reporting a higher overall PFBS score, suggesting that they were experiencing more objective and subjective burden. However, Chinese caregivers did not report a higher amount of burden within each present behaviour, which could be due either to a tendency within the Chinese group to simply report more behaviours as being present, or because the test lacked sufficient power to reveal this particular effect.

Finally, Chinese caregivers had a more negative conceptualization of mental illness than did Euro-Canadian caregivers. Chinese caregivers were particularly more likely to endorse the practice of keeping mental illness a secret from others, as well as withdrawal from individuals with mental illness (although it should not be assumed that this latter tendency characterized the caregivers' relationships with their own relatives). Both of these findings suggest that, as predicted elsewhere (e.g. Lin \& Lin, 1981; Shon \& Ja, 1982), Chinese caregivers were more affected by the stigma of mental illness than were Euro-Canadian caregivers.

Many of these findings are consistent with prior studies of Chinese culture and mental health. For example, a pattern of treatment delay, combined with a tendency to keep mental illness a secret, is consistent with Kirmayer's assertions that Chinese families are reported to be more likely to try to shield the ill family member from the rest of the community, leading to treatment delay (Kirmayer, 1989). Similarly, prior research on a Canadian sample by Lin and colleagues (1978) found that tolerance for psychotic symptoms by Chinese families was higher than by Euro- Canadian families during the early stages of the illness. Acceptance declined 
and withdrawal began, however, after the initiation of extrafamilial treatment seeking (Lin, Tardiff, Donetz, \& Goresky, 1978). Given that all of the participants in our study were at this later stage, these findings are compatible with the reports of higher burden and greater endorsement of withdrawal found in our sample.

Further insight into the meaning of this pattern of results can be found by reviewing some reported central features of Chinese culture. Wu and Tseng (1985) have identified five important elements integral to Chinese communities worldwide: (a) family and collective responsibility, (b) emphasis on the parent-child bond, (c) social interactions in a personal network, (d) emphasis on emotional control and morality, and (e) valuation of education and achievement. Psychotic mental illness usually involves social, occupational, and emotional difficulties, thus leading to disruption of several, if not all, of these cultural values. In particular, because the family is seen as the focal point for socialization of members, the asis on collective responsibility leads to a belief that mental illness is a family problem, with all members at least partially responsible for its occurrence. As a result of these internal and external pressures, Chinese caregivers may prefer to deal with mental illness within the family for as long as possible. Western families, in contrast, may be more likely to perceive psychotic mental illness as requiring medical or psychiatric attention, and thus feel responsible for ensuring that their ill relative receive such treatment.

Another contributing factor to the results found in this study may be the quality of care, perceived or actual, offered by Western mental health facilities to minority groups. Investigators have found deficiencies in the delivery of mental health services to Chinese in North America, including differential forms of assessment and treatment, therapist preferences for client characteristics that place minorities at a disadvantage, high premature termination rates, and overall ineffectiveness (Leong, 1986; Sue, 1992). Our finding that Chinese take longer than Euro-Canadians to present for treatment may be part of an overall tendency to avoid discriminatory treatment from Western mental health facilities. However, such an explanation cannot explain all of the variance observed, as our measure of treatment delay included all potential sources of external help, both traditional and Western.

The association of Chinese ethnicity with the immigration experience should also be explored, in order to better understand the relative contributions of ethnicity and acculturation. Immigration is often associated with social isolation, language barriers, and a lesser understanding of how to work within the sociopolitical system (Dion \& Dion, 1996), and could thus be associated with a longer waiting period after onset of psychosis before Western-style medical care is sought. Similarly, the extent of acculturation following immigration has been associated both with psycho- logical adjustment and with attitudes towards counselling services (for a review see Dion \& Dion, 1996). For example, Atkinson and Gim (1989) explored the relationship of help-seeking attitudes with ethnicity, gender and acculturation, and found that high acculturation was the best predictor of positive psychiatric help-seeking attitudes.

To address some of the shortcomings of the present study, future research should include a larger sample, particularly from the Chinese group. Moreover, it may be useful to supplement future findings with qualitative approaches, which can often provide a rich picture of experiences, burden, and culture. Nevertheless, the results of this study indicate that, despite the low variability in the natural course of schizophrenia, ethnic differences may be important in mediating the experience of psychosis both for the affected individual and for his or her family.

\section{Acknowledgments}

The authors would like to thank Luke Conway, Joy Harrison, Jennifer Ritsher, Roger Tweed, and Jiahui ZhangWong for their helpful comments on earlier drafts of this manuscript. In addition, we gratefully acknowledge the help of the nursing staff and psychiatrists of the First Episode Psychosis program.

The study was supported by a National Alliance for Research on Schizophrenia and Depression (NARSAD) Young Investigator Award to the second author. Preparation of this paper was also facilitated by a research grant from the Social Sciences and Humanities Research Council of Canada to the third author.

\section{References}

American Psychiatric Association (1994). Diagnostic and statistical manual of mental disorders (4th ed.). Washington, DC: American Psychiatric Association. Atkinson, D. R. \& Gim, R. H. (1989). Asian-American cultural identity and attitudes toward mental health services. Journal of Counseling Psychology, 36, 209-212.

Aviram, V. (1990). Community care of the seriously mentally ill: Continuing problems and current issues. Community Mental Health Journal, 26, 69-85.

Beiser, M., Erickson, D., Fleming, J. A. E., \& Iacono, W. G. (1993). Establishing the onset of psychotic illness. American Journal of Psychiatry, 150, 1349-1354.

Blishen, B. R., Carroll, W. K., \& Moore, C. (1987). The 1981 socioeconomic index for occupations in Canada. Canadian Review of Sociology and Anthropology, 24, 467-488.

Cohen, J. (1988). Statistical power analysis for the behavioral sciences (2nd ed.). Hillsdale, NJ: Lawrence Erlbaum Associates.

Dion, K. L., \& Dion, K. K. (1996). Chinese adaptation to foreign cultures. In M. H. Bond (Ed.), The handbook of Chinese psychology (pp. 457-478). Hong Kong: Oxford University Press. 
Hatfield, A. (1986). Semantic barriers to family and professional collaboration. Schizophrenia Bulletin, 12, 325-327.

Hoenig, J., \& Hamilton, M. (1965). The desegregation of the mentally ill. London: Routledge \& Kegan Paul.

Howell, D. C. (1992). Statistical methods for psychology, 3rd edition. Belmont, CA: Wadsworth.

Kirmayer, L. J. (1989). Cultural variations in the response to psychiatric disorders and emotional distress. Social Science $\mathcal{E}$ Medicine, 29, 327-339.

Lefley, H. (1987). Impact of mental illness in families of mental health professionals. Journal of Nervous and Mental Disease, 175, 613-619.

Leong, F. T. (1986). Counseling and psychotherapy with Chinese-Americans: Review of the literature. Journal of Counseling Psychology, 33, 196-206.

Levene, J. E., Lancee, W. J., \& Seeman, M. V. (1996). The perceived family burden scale: Measurement and validation. Schizophrenia Research, 22, 151-157.

Lin, T.-Y., \& Lin, M. C. (1981). Love, denial and rejection: Responses of Chinese families to mental illness. In A. Kleinman \& T. Y. Lin (Eds.), Normal and abnormal behaviour in Chinese societies. Dordrecht, the Netherlands: D. Reidel.

Lin, T-Y., Tardiff, K., Donetz, G., \& Goresky, W. (1978). Ethnicity and patterns of help-seeking. Culture, Medicine and Psychiatry, 2, 3-13.

Link, B. G. (1987). Understanding labeling effects in the area of mental disorders: An assessment of the effects of expectations of rejection. American Sociological Review, 52, 96-112.

Link, B. G., Mirotznik, J., \& Cullen, F. T. (1991). The effectiveness of stigma coping orientations: Can negative consequences of mental illness labeling be avoided? Journal of Health and Social Behavior, 32, 202-320.

Loukissa, D. A. (1995). Family burden in chronic mental illness: A review of research studies. Journal of Advanced Nursing, 21, 248-255.

MacCarthy, B., Lesage, A., Brewin, C. R., Brugha, T. S., Mangen, S., \& Wing, J. K. (1989). Needs for care among the relatives of long-term users of day care: A report from the Camberwell High Contact Survey. Psychological Medicine, 19, 725-736.

Mueser, K. T., Webb, C., Pfeiffer, M., Gladis, M., \& Levinson, D. F. (1996). Family burden of schizophrenia and bipolar disorder: Perceptions of relatives and professionals.
Psychiatric Services, 47, 507-511.

Rolland, J. (1987). Family illness paradigms: Evolution and significance. Family Systems Medicine, 5, 482-503.

Shon, S. P., \& Ja, D. Y. (1982). Chinese families. In M. McGoldrick, J. K. Pearce, \& J. Giordano (Eds.), Ethnicity and family therapy. New York: Guilford.

Siegel, K., Raveis, V., Mor, V., \& Houts, P. (1991). The relationship of spousal caregiver burden to patient disease and treatment-related conditions. Annals Oncology, 2, 511-516.

Smith, J., Birchwood, M., Cochrane, R., \& George, S. (1992). The needs of high and low expressed emotion families: A normative approach. Social Psychiatry and Psychiatric Epidemiology, 28, 11-16.

Spitzer, R. L., Williams, J. B. W., \& Gibbon, M. (1987). Structured Clinical Interview for DSM-III-R (SCID). New York: New York State Psychiatric Institute Biometrics Research Department.

Sue, S. (1992). Ethnicity and mental health: Research and policy issues. The Journal of Social Issues, 48(2), 187-205.

Wu, D. Y. H., \& Tseng, W. S. (1985). Introduction: The characteristics of Chinese culture. In W. S. Tseng \& D. Y. H. Wu (Eds.), Chinese culture and mental health. Orlando, FL: Academic.

Yeh, M., Takeuchi, D. T., \& Sue, S. (1994). ChineseAmerican children treated in the mental health system: A comparison of parallel and mainstream outpatient service centers. Journal of Clinical Child Psychology, 23, 5-12.

Zhang-Wong, J., Beiser, M., Zipursky, R., \& Bean, G. (1998). An investigation of ethnic and gender differences in the pharmacodynamics of haloperidol. Psychiatry Research, 81, 333-339.

ANDREW G. RYDER conducted this research while a student in the Department of Psychology, University of Toronto, and is now a $\mathrm{PhD}$ clinical psychology student in the Department of Psychology, University of British Columbia. Address: Department of Psychology, University of British Columbia, 2136 West Mall, Vancouver, BC, Canada, V6T 1Z4; Fax: (604) 822-6923; E-mail: agryder@interchange.ubc.ca.

GRAHAM BEAN is Assistant Professor, Department of Psychiatry, University of Toronto and Research Scientist, Neurogenetics Unit, Clarke Institute of Psychiatry. Address: Neurogenetics Unit, Clarke Institute of Psychiatry, 250 College Street, Toronto, Ontario, Canada, M5T 1R8.

KENNETH L. DION is Professor of Social Psychology, Department of Psychology, University of Toronto. 